\title{
On Optimisation of Environmentally Friendly Aircraft Engine Cycles
}

\author{
Cesar Celis ${ }^{1}$, Vishal Sethi², Riti Singh², Pericles Pilidis²
}

\begin{abstract}
In this work, the Techno-economic Environmental Risk Analysis framework, a multi-disciplinary optimisation tool developed by Cranfield University, is utilised in conjunction with an in-house optimiser to carry out aircraft engine cycle optimisation processes. The central point here is the evaluation of the capabilities of the in-house optimiser for performing this type of optimisation processes. Simplifying hypotheses are thus considered when both defining the aircraft flight trajectory and modelling the different engine configurations analysed. Accordingly, several optimum engine cycles minimising separately three objective functions, (i) specific fuel consumption in cruise, (ii) fuel burned, and (iii) oxides of nitrogen emitted, are determined. The cycle optimisation processes carried out yield results reflecting the general trends expected when optimising according to these objective functions. It follows then that the in-house optimiser is suitable for carrying out gas turbine power plant optimisation processes. It is expected that this optimiser be utilised in future for both optimising the preliminary design of gas turbine engines and determining optimum and "greener" aircraft engine cycles.
\end{abstract}

KEYWORDS: Engine cycle optimisation, Aircraft emissions, Environmental impact.

\section{INTRODUCTION}

There is an increasing concern about the current natural resource consumption trends and the impact of human activities on the environment. This is justified as anthropogenic pollutants have reached very high levels. It is imperative, therefore, that major targeted investments be made into economical and reliable environmental friendly power plant solutions. Currently, there is a large variety of promising power and propulsion concepts that are being proposed to mitigate or eliminate carbon dioxide and other emissions. These include new aircraft engines featuring conventional and novel cycles, nuclear power plants, carbon capture schemes, renewable energy sources (including onshore and offshore wind and wave energy systems), and many others. The systems envisaged are larger and more complex than those corresponding to the current state of the art. Inevitably, this will result in an increase in the power plant initial cost, a greater difficulty to meet reliability and availability requirements, and more complex operating procedures. Furthermore, the combined objectives of economic and environmental performance make the decision process much more difficult. All these challenges will need to be addressed when evaluating investment performance. In addition, for many of these concepts, there is little or no relevant operating experience. So, the selection of the best candidates to pursue for investment becomes very difficult and uncertain, and the risks are high. The Techno-economic Environmental Risk Analysis (TERA) framework/tool provides an answer to some of these questions. TERA can inform investors, legislators, and other stakeholders of the best investments needed to achieve a cleaner environment, and, hopefully, to accelerate the accrual of benefits from advanced low carbon power and propulsion systems.

1.Pontifícia Universidade Católica do Rio de Janeiro - Departamento de Engenharia Mecânica - Rio de Janeiro/RJ - Brazil 2.Cranfield University - School of Engineering - Cranfield/Bedfordshire - United Kingdom.

Author for correspondence: Cesar Celis | Departamento de Engenharia Mecânica - Pontifícia Universidade Católica do Rio de Janeiro | Rua Marquês de São Vicente 225 | CEP: 22.451-900 - Rio de Janeiro/RJ - Brazil | Email: cesar.celis@puc-rio.br

Received: 08/15/2014 | Accepted: 02/11/2015 
TERA (Ogaji et al., 2007, 2009, 2010; Kyprianidis et al., 2008, 2009; Khan et al., 2009; Celis et al., 2008; Di Lorenzo et al., 2007), an idea conceived and developed by Cranfield University, is essentially a multi-disciplinary optimisation tool for quantifying risks and for comparing and ranking competing power plant schemes on a formal and consistent basis. TERA involves the use of several high fidelity preliminary design modules integrated with commercial/ in-house optimisers. TERA was primarily developed for determining optimum power plants for a given application. Recently, however, TERA has been also utilised for performing efficient aircraft trajectory multi-disciplinary optimisation processes accounting for physical and Air Traffic Control constraints (Celis et al., 2014). TERA has evolved over the years and it is currently utilised mainly as a tool to explore engine design spaces at a quick and efficient way. Nevertheless, the TERA philosophy is applicable to the different fields in which gas turbines are utilised (land, air, and sea). TERA as a tool is constantly updated and adapted with new algorithms and mathematical models which help to increase the level of reliability of the results obtained. The most important part of TERA is a detailed and rigorous thermodynamic representation of power plants, i.e. the engine performance module. Coupled to this module, other modules that model different disciplines, such as aircraft performance, economics, emissions, noise, weight etc., are utilized within the TERA framework. In this particular work, TERA has been utilised in conjunction with an in-house optimiser, i.e. oPtimisatiOn aLgorithms librarY for PHysical complEx MUlti-objective problemS ("Polyphemus") (Celis et al., 2014), to carry out aircraft engine cycle optimisation processes. Polyphemus utilises genetic algorithms-based optimisation routines which do not use specific knowledge of the optimisation problem domain. This is a key-aspect as it allows that Polyphemus be both model and problem independent, satisfying in this way one of the main requirements of optimisation processes involving multi-model integration.

Shortly, Polyphemus has been implemented by using Java as the main programming language. Its core has been developed following the basic structure of the "SGA Java V1.03" by Hartley (Hartley, 1998), a Java implementation of the "simple GA" by Goldberg (1989). Nevertheless, the original model has been recoded and extensively modified to both adapt it to engineering design optimisation problems and maximise its performance. The main modifications introduced relate to the improvement of the optimisation performance, through an adaptation to the application domain (engineering design), and improvements in both the technique (introduction of concepts such as elitism, steady state replacement and fitness scaling) and the genetic operators (crossover, mutation and selection) utilised during the optimisation process. Polyphemus uses a unique optimisation method based on Wienke's idea of target vector optimisation (Wienke et al., 1992). Following this approach, designers can define, for each parameter, a target to be attained, a range within which this parameter should remain, and the requirement to maximise or minimise the given parameter. The quality of the design is then determined from the achievement of the targets, the possibility of violation of ranges, and the optimisation of the selected parameters. This approach enables designers to have total control over the optimisation process with neither having to know very much about the optimisation algorithms, nor having to devise a fitness function (Rogero and Rubini, 2003). Additional details of the Polyphemus optimiser can be found in Celis (2010) and Celis et al. (2009a, 2014).

The aircraft engine cycle optimisation processes carried out in this work focused mainly on the evaluation of the capabilities of Polyphemus for performing this type of optimisation processes. For the determination of optimum engine cycles then, simplifying hypotheses were considered when (i) defining the aircraft flight trajectory, and (ii) modelling the different engine configurations analysed. This afforded greater visibility on the characteristics of the Polyphemus performance when assessing results. This would have been more difficult if all complexities involving this type of optimisation processes had been considered. Accordingly, several optimum engine cycles minimising separately three objective functions, (i) specific fuel consumption (SFC) in cruise, (ii) fuel burned, and (iii) oxides of nitrogen (NOx) emitted, were determined. The main results of these optimisation processes are summarised here.

\section{ENGINE CYCLE OPTIMISATION CASE STUDIES}

General aspects about the engine cycle optimisation processes carried out in this work are briefly described in this section. A particular emphasis is placed on the adopted optimisation strategy, the TERA computational models utilised, as well as 
the main parameters used as design variables, constraints, and optimisation criteria during the optimisation processes.

\section{OPTIMISATION STRATEGY}

Generally, two broad optimisation strategies can be adopted for the optimisation of the preliminary design of aircraft/engine configurations. Firstly, both the aircraft/engine configuration and its associated flight profile (flight trajectory) can be optimised simultaneously (Antoine and Kroo, 2005; Antoine et al., 2004; Diedrich et al., 2006). In this approach, the optimisation of the flight profile is usually treated as a sub-optimisation process. Accordingly, for each aircraft/engine configuration evaluated (potential optimum design), an optimum flight profile according to given criteria is determined. Due to the simultaneous optimisation of both aircraft/engine configuration and flight trajectory, this approach generally yields more representative results than the second simplified one indicated next.

The second optimisation strategy is a simplification of the first one and involves the optimisation of the aircraft/ engine configuration considering that the aircraft flight profile is fixed. The main advantage of this second approach is that the computational time involved in the process is largely reduced. This reduction in computational time is proportional to several aspects including fidelity of the computational models, type of optimisation technique, complexity of the trajectory optimisation problem, and flight profile discretisation level. In the engine cycle optimisation processes performed in this work, this second optimisation strategy was utilised. The main reason behind this choice is the fact that natural limitations in computational time were present during the development of this work. This was supported by the fact that optimum trajectories (for a fixed aircraft/engine configuration) had already been determined (Celis, 2010). The main parameters characterising the fixed aircraft trajectory utilised in the optimisation processes described in this work are highlighted in Table 1.
In Table $1, h$ represents flight altitude; $M$, Mach number; EAS, equivalent airspeed; and $R$, range. The subscripts $i$ and $f$ refer to, respectively, the start and end of the flight trajectory segments. This aircraft trajectory corresponds to a three-objective (flight time, fuel burned, and $\mathrm{NO}_{\mathrm{x}}$ emitted) optimised trajectory obtained from multi-objective optimisation processes carried out in a previous work (Celis, 2010). In short, this trajectory can be described as follows - $1^{\text {st }}$ segment: climb at constant EAS (124.5 m/s) from 1,500 ft (457 m) up to 10,000 ft (3,048 m); $2^{\text {nd }}$ segment: EAS acceleration at 10,000 ft (level flight) to $134.1 \mathrm{~m} / \mathrm{s}$ EAS; $3^{\text {rd }}$ segment: climb at constant EAS $(134.1 \mathrm{~m} / \mathrm{s})$ up to an altitude where (cruise) $M$ is about 0.8 , i.e. $10,411 \mathrm{~m} ; 4^{\text {th }}$ and $5^{\text {th }}$ segments: level flight cruise at constant $M(0.8) ; 6^{\text {th }}$ segment: descent at constant EAS $(134.1 \mathrm{~m} / \mathrm{s})$ to $10,000 \mathrm{ft}(3,048 \mathrm{~m}) ; 7^{\text {th }}$ segment: EAS deceleration at 10,000 ft (level flight) to $128.6 \mathrm{~m} / \mathrm{s}$ EAS; and finally, $8^{\text {th }}$ segment: descent at constant EAS $(128.6 \mathrm{~m} / \mathrm{s})$ from $10,000 \mathrm{ft}(3,048 \mathrm{~m})$ to $1,500 \mathrm{ft}(457 \mathrm{~m})$. This flight trajectory has been considered fixed and has been used as the baseline trajectory for performing the engine cycle optimisation processes described here.

\section{COMPUTATIONAL MODELS}

In the optimisation processes, only three TERA computational models, i.e. the aircraft performance simulation model (APM), the engine performance simulation model (TurboMatch), and the emissions prediction model (Hephaestus), have been utilised. Figure 1 illustrates the general arrangement of these models, as well as the different parameters exchanged among them. The APM (Long, 2009) is a generic tool able to determine flight path performance for a given aircraft design. APM uses steady state performance equations to resolve aerodynamic lift and drag and determines the thrust required for a given kinematic flight state. In order to easily identify the behaviour of Polyphemus, airspeed limitations, such as critical $M$, never-exceed speed, and wave drag at transonic $M$, have not been implemented in the

Table 1. Fixed reference aircraft trajectory - characteristic parameters.

\begin{tabular}{|c|c|c|c|c|c|c|c|}
\hline Segment (no.) & $h_{i}[\mathrm{~m}]$ & $h_{f}[\mathrm{~m}]$ & $M_{i}[-]$ & $M_{f}[-]$ & $\mathrm{EAS}_{i}[\mathrm{~m} / \mathrm{s}]$ & $\mathrm{EAS}_{f}[\mathrm{~m} / \mathrm{s}]$ & $\boldsymbol{R}[\mathrm{km}]$ \\
\hline 1 & 457 & 3,048 & -- & -- & 124.5 & 124.5 & 20 \\
\hline 2 & 3,048 & 3,606 & -- & -- & 124.5 & 134.1 & 10 \\
\hline 3 & 3,606 & 10,411 & -- & -- & 134.1 & 134.1 & 160 \\
\hline 4 & 10,411 & 10,411 & 0.8 & 0.8 & -- & -- & 230 \\
\hline 5 & 10,411 & 10,411 & 0.8 & 0.8 & -- & -- & 230 \\
\hline 6 & 10,411 & 3,659 & -- & -- & 134.1 & 134.1 & 140 \\
\hline 7 & 3,659 & 3,048 & -- & -- & 134.1 & 128.6 & 20 \\
\hline 8 & 3,048 & 457 & -- & -- & 128.6 & 128.6 & 70 \\
\hline
\end{tabular}


model. Since APM uses end-points to compute performance, the user must declare a trajectory segment in terms of ground range and altitude intervals whereby a constant flight path angle is then defined. Flight conditions are then assumed to be constant over that segment. The aircraft modelled in this work corresponds to a typical mid-sized, single-aisle, twin turbofan airliner with a maximum take off weight of about $72,000 \mathrm{~kg}$ and a seating capacity of about 150 passengers.

The performance of the engines was simulated using TurboMatch (Palmer, 1999), the in-house Cranfield University gas turbine performance code that has been refined and developed over a number of decades. TurboMatch performance simulations range from simple steady state (design and off-design point) to complex transient performance computations. Finally, the gaseous emission predictions have been performed using the Cranfield University emissions prediction software Hephaestus. An integral part of Hephaestus constitutes the emissions prediction model described in Celis et al. (2009b), which follows an approach based on the use of a number of stirred reactors for modelling combustion chambers and estimating the level of pollutants emitted from them. Additional details of these computational models can be found in Celis (2010) and Celis et al. (2009a).

\section{DESIGN VARIABLES}

The following cycle parameters have been utilised in this work as main design variables: overall pressure ratio (OPR), bypass ratio (BPR), and turbine entry temperature (TET). These cycle parameters were chosen because they characterise the design of any turbofan engine - the particular type of engine (cycle) optimised in this work. In the optimisation processes, however, OPR was not directly used as a design variable. Instead, it was represented by the other three parameters that characterise OPR in two- or three-spool (turbofan) engines: fan pressure ratio (FPR), booster or intermediate pressure compressor pressure ratio (IPCPR), and high pressure compressor pressure ratio (HPCPR).

Top of climb (TOC) has been utilised in this work as the design point (DP) condition of the engines. Consequently, due to the aircraft trajectory flight phase involving take off (TO) was not included in the analyses carried out, an additional design variable, TET at TO, was utilised in the optimisation processes. This last design variable was included in order to estimate parameters (detailed in the next section) that help to verify whether (or not) a given engine design satisfies the engine requirements at TO (off design, OD) conditions. Table 2 summarises then the design variables (and the ranges of permissible values considered) utilised in the different case studies analysed in this work.

\section{IMPLICIT CONSTRAINTS}

During the optimisation, the following implicit constraints were imposed: thrust ratio (TR), compressor delivery temperature (CDT) at TO (CDTTO), height of blade (HBL) of the high pressure compressor (HPC) last stage at TO (HBLTO), and net thrust (FN) at DP and TO. TR, the ratio of TO thrust to cruise thrust (TOC in this work), is usually a requirement dictated by the airframe on which the engine is installed. Even so, in practice, some adjustments in TO thrust are possible as TO field length

Table 2. Summary of design variables.

\begin{tabular}{|c|c|c|c|}
\hline Name & Unit & Lower bound & Upper bound \\
\hline FPR & {$[--]$} & 1.1 & 1.9 \\
\hline IPCR & {$[--]$} & 2.0 & $5.0\left(15.0^{*}\right)$ \\
\hline HPCPR & {$[--]$} & $5.0\left(2.0^{*}\right)$ & $20.0\left(15.0^{*}\right)$ \\
\hline BPR & {$[--]$} & 2.0 & 15.0 \\
\hline TET & {$[\mathrm{K}]$} & 1,200 & 1,800 \\
\hline TET TO & {$[\mathrm{K}]$} & 1,200 & 1,900 \\
\hline
\end{tabular}

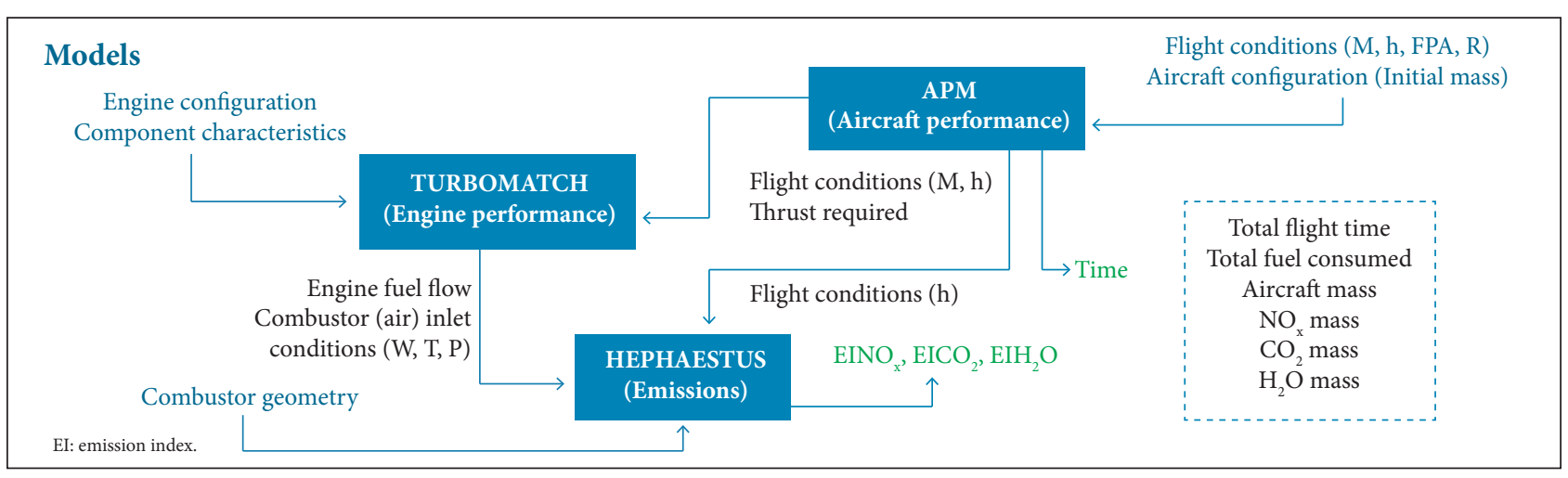

Figure 1. Computational model configuration. 
varies. TR was used as a constraint in this work in order to guarantee that an optimised engine is able to provide the required TO thrust. Thus, a TR lower limit of 4.5 was utilised. This value reflects the performance of a typical turbofan, powering a midsized, single-aisle, twin turbofan airliner (maximum take off weight $\approx 72,000 \mathrm{~kg}$ ) and delivering thrust levels of about $25 \mathrm{kN}$ at TOC and $112.5 \mathrm{kN}$ at TO. The CDTTO constraint reflects, in turn, the level of technology, in terms of material capability, of the last stages of the HPC. This is one of the main limiters to the level of OPR that can be achieved in conventional turbofan engines. Excessive values of CDTTO would require the use of especial materials for the disc and blades of the HPC rear stages (which could increase the engine weight). In addition, high CDTTO values could also cause cooling problems due to the high temperatures of the cooling flows used for cooling the high pressure turbine (HPT) components. In the optimisation, an upper limit for the CDTTO of $950 \mathrm{~K}$ was considered.

Constant overall engine/nacelle dimensions were considered during the optimisation processes because of some limitations in the APM. This was made possible through the use of a fixed overall (engine) inlet mass flow rate at DP. Thus, high values of OPR and BPR will require eventually small blades at the rear of the HPC, which are known to be characterised by high aerodynamic losses because of the low Reynolds numbers, and the comparatively thick boundary layers on the annulus walls and high tip clearances (Whellens, 2003). In addition, because of their size, small blades may present manufacturing problems. It was necessary thus to constraint the HBLTO values. In this work, this parameter was estimated using the "swallowing function" (Eq. 1) and assuming a flow $M$ and a compressor hub/ tip ratio at the HPC delivery section of 0.3 and 0.9 , respectively:

$$
\frac{W \sqrt{T}}{A P}=M\left(\sqrt{\frac{\gamma}{R_{g}}}\right)\left[1+\frac{(\gamma-1)}{2} M^{2}\right]^{\frac{-(\gamma+1)}{2(\gamma-1)}}
$$

where:

$W$ : mass flow rate; $T$ : temperature; $P$ : pressure; $A$ : flow area; $M$ : Mach number; $R_{g}$ : specific gas constant; $\gamma$ : ratio of specific heats of the gas. In the computations, a lower limit for the HBLTO of $15 \mathrm{~mm}$ was utilised.

Table 3 summarises the implicit constraints used in the different engine cycle optimisation processes performed, unless otherwise explicitly indicated. In this Table, the FN (at DP and TO) implicit constraints are marked with asterisks in order to highlight that they were used only in some of the optimisation processes carried out. When just the first three constraints indicated in Table 3 are utilised, the fact that the aircraft is able to fly the reference flight trajectory, using a given engine design, is the only criterion determining (internally) the validity of the design. Consequently, it is recognised that some variations in FN at DP and/or TO may exist as a result of the optimisation processes. However, considering the main purpose of the optimisation processes performed, this was not seen as a critical issue. It is worth emphasising that these parameters (i.e. FN at DP and/or TO) were not initially constrained mainly because of the gradual approach, in terms of addition of complexities (e.g. number of implicit constraints), followed in this work.

Table 3. Summary of implicit constraints.

\begin{tabular}{|c|c|c|}
\hline No. & Constraint & Limit[s] \\
\hline 1 & TR & $\geq 4.5$ \\
\hline 2 & CDTTO & $\leq 950 \mathrm{~K}$ \\
\hline 3 & HBLTO & $\geq 15 \mathrm{~mm}$ \\
\hline 4 & FN & $\pm 1 \%$ of $25.4 \mathrm{kN}^{*}$ \\
\hline 5 & FN TO & $\pm 1 \%$ of $121.4 \mathrm{kN}^{* *}$ \\
\hline
\end{tabular}

* Used in the computations of ICR engines; **used in the computations carried out in the "Further Results" section only.

\section{PERFORMANCE PARAMETERS}

Performance parameters are used for establishing the comparison criterion of several acceptable designs which ultimately allows the determination of the best design among many acceptable ones. This criterion, with respect to which the design is optimised, when expressed as a function of the design variables, is known as the criterion or merit or objective function. In this work, only single-objective engine cycle optimisation processes were carried out. This means that only single-objective functions were utilised. Since they have a direct influence on the environmental impact of commercial aircraft operations, two of the three objective functions utilised in this work were fuel burned and $\mathrm{NO}_{\mathrm{x}}$ emissions. The third objective function involved the specific fuel consumption in cruise (cruise SFC), which was computed averaging the SFC corresponding to segments 4 and 5 considered as cruise in the reference aircraft trajectory utilised ( $c f$. Table 1 ). Several optimum engine cycles minimising separately these three objective functions, cruise SFC, fuel burned, and $\mathrm{NO}_{\mathrm{x}}$ emitted, were thus determined and the main results are summarised in this work.

\section{ENGINE CYCLES}

In this work, three engine cycles corresponding to three different aircraft engines (with separate exhausts), i.e. two-spool 
turbofan engine, three-spool turbofan engine, and (two-spool) Intercooled Recuperated Turbofan (ICR) engine, were studied. These engines were selected because they represent potential engines that could be eventually utilised in regional aircraft configurations similar to that analysed in this work. Table 4 details the main parameters characterising the engines used as reference or baseline during the optimisation processes. In this table, $W$ represents the overall (engine) inlet (air) mass flow rate. Table 4 shows that the engine condition used as DP condition corresponded to TOC (10,668 m [35,000 ft], $M=0.8$, International Standard Atmosphere - ISA), implying, in this way, that TO (sea level $[\mathrm{S} / \mathrm{L}], M=0.0, \mathrm{ISA}+30^{\circ} \mathrm{C}$ ) was treated as an $\mathrm{OD}$ point engine condition.

For the design of the two-spool baseline engine (Table 4), an iterative process involving engine simulations at DP and OD point conditions was utilised in order to match the performance of the engine model with data obtained from the public domain for an aircraft engine (high BPR, two-spool turbofan engine with separate exhausts) used in similar applications. Similarly, for the design of the three-spool and ICR baseline engines, iterative processes involving engine simulations at DP and OD point conditions were used in order to match the thrust requirements of the engine models with those corresponding to the two-spool baseline engine initially designed. During the design of these baseline engines, when necessary, educated guesses were made for some characteristic parameters (component efficiencies, bleeding flows, pressure losses etc.), which were required for the modelling of the engines. Accordingly, in order to take into account the state of the

Table 4. Baseline engine characteristic parameters.

\begin{tabular}{|c|c|c|c|c|}
\hline \multicolumn{5}{|c|}{ DP: $\operatorname{TOC}(10,668 \mathrm{~m}[35,000 \mathrm{ft}], \mathrm{M}=0.8, \mathrm{ISA}]$} \\
\hline Parameter & Unit & Two-spool & Three-spool & ICR \\
\hline W & {$[\mathrm{kg} / \mathrm{s}]$} & 180.0 & 180.0 & 180.0 \\
\hline BPR & {$[--]$} & 5.46 & 5.46 & 5.46 \\
\hline FPR & {$[--]$} & 1.80 & 1.70 & 1.70 \\
\hline IPCPR & {$[--]$} & 1.81 & 4.38 & 1.92 \\
\hline HPCPR & {$[--]$} & 10.0 & 4.38 & 10.0 \\
\hline OPR & {$[--]$} & 32.6 & 32.6 & 32.6 \\
\hline TET & {$[\mathrm{K}]$} & 1,340 & 1,412 & 1,280 \\
\hline $\mathrm{FN}$ & {$[\mathrm{kN}]$} & 25.1 & 25.2 & 25.4 \\
\hline SFC & {$[\mathrm{mg} / \mathrm{Ns}]$} & 17.0 & 17.1 & 17.2 \\
\hline \multicolumn{5}{|c|}{ OD: $\mathrm{TO}\left(\mathrm{S} / \mathrm{L}, \mathrm{M}=0.0, \mathrm{ISA}+30^{\circ} \mathrm{C}\right)$} \\
\hline Parameter & Unit & Two-spool & Three-spool & ICR \\
\hline TET TO & {$[\mathrm{K}]$} & 1,600 & 1,655 & 1,510 \\
\hline FN TO & {$[\mathrm{kN}]$} & 121.4 & 121.7 & 120.4 \\
\hline
\end{tabular}

art associated with the design of the main components of aircraft engines, namely compressors and turbines, appropriate component polytropic efficiencies which attempt to reflect the current level of technology in this field were assumed. These efficiencies, which remained constant at DP during the optimisation, were equal to: 0.93 in the particular case of the fan, 0.91 for the intermediate pressure compressor (IPC) and HPC, 0.88 for the HPT, 0.89 for the intermediate pressure turbine (IPT, used only for three-spool configurations), and 0.90 for the low pressure turbine (LPT).

It is relevant to note that the main purpose of the engine cycle optimisation processes carried out in this work was the evaluation of the capabilities of Polyphemus to perform these types of processes. Simplifications were thus introduced into all optimisation processes when both defining the aircraft flight trajectory (e.g. small number of trajectory segments, limited number of flight phases, standard atmospheric conditions etc.) and modelling the different engine configurations (e.g. constant nacelle/engine dimensions and weight, limited number of map of characteristics for compressors and turbines, simplified algorithms for pollutant formation etc.). It follows then that, when analysing the results obtained from the optimisation processes, it is considered that general trends are more reliable than absolute values.

In the engine cycle optimisation processes performed, the following four main hypotheses were considered:

- Aircraft flight altitudes and speeds are constant.

- Aircraft configuration (dimensions, weight etc.) is fixed. In other words, the aircraft is not resized during the optimisation processes. This hypothesis was used mainly because the APM can handle only fixed aircraft/ engine configurations.

- $\quad$ Aircraft engine (nacelle + engine) dimensions and weight remain constant regardless of the variations in the engine thermodynamic cycle characteristic parameters. This was hypothesised partially because engine weight models were not used in this work.

- Total aircraft weight (aircraft empty weight + engine weight + fuel on-board) at the beginning of the flight profile is constant. It implies that fuel on-board is enough for flying the flight profile using any engine design. This hypothesis avoids the use of iterative processes during the optimisation.

Accordingly, three different cases studies, each of them involving the optimisation of a given aircraft engine cycle, were separately analysed. A brief description of these case studies is presented below: 
- $\quad$ Case 1: two-spool turbofan optimisation. In the first case study, engine cycle optimisation processes involving a twospool turbofan engine with separate exhausts were carried out. The parameters used as design variables in this case corresponded to those described in Table 2. As implicit constraints, in turn, only the first three parameters indicated in Table 3 (TR, CDTTO, and HBLTO) were utilised.

- Case 2: three-spool turbofan optimisation. Optimisation processes involving a three-spool turbofan engine cycle with separate exhausts were performed in this case. The design variables and constraints were the same as in the first case study. The only difference related to the ranges of permissible values of two of the design variables (IPCPR and HPCPR), which were slightly modified. This was done in order to reflect the fact that these parameters may have a similar order of magnitude.

- Case 3: intercooled recuperated turbofan optimisation. In the third case study, the thermodynamic cycle of an ICR two-spool turbofan engine with separate exhausts was optimised. In addition to the design variables and constraints used in the first two case studies, and additional implicit constraint, i.e. FN at DP, was imposed in this case (cf. Table 3 ). It was additionally assumed that: (i) the use of intercooling and recuperation systems does not imply variations in the engine dimensions; (ii) the ICR engine is $50 \%$ heavier than a conventional one used for similar purposes; (iii) the heat exchangers produce additional pressure losses of $3 \%$ (each one); and (iv) the heat exchangers' effectiveness is about $90 \%$. These assumptions attempted to reflect a typical scenario where ICR engines might be utilised.

In addition to the optimisation processes performed as part of the case studies mentioned, in order to compare the three engine cycles analysed in these cases, other optimisation processes were also carried out. In these processes, for allowing a fair comparison among the engine cycles, additional implicit constraints were imposed when required. These constraints related to FN at DP and TO. As indicated in Table 3, the ranges of permissible values in which these two constraints were allowed to vary corresponded to $\pm 1 \%$ of their corresponding nominal values. These nominal values were taken, in turn, from the respective values of FN at DP and TO (cf. Table 4; DP: $25.1 \mathrm{kN}$, TO: $121.4 \mathrm{kN}$ ) associated with the baseline two-spool engine, which was also considered as baseline engine in this comparison process. The main results obtained from this comparison process are also presented and discussed in this work.

\section{RESULTS AND DISCUSSIONS}

The main results of the optimisation processes corresponding to the three case studies indicated in the Engine Cycles section are summarised in this section.

\section{CASE 1: TWO-SPOOL TURBOFAN OPTIMISATION}

The results obtained from the optimisation processes carried out in this first case study are summarised in Fig. 2. More specifically, the main parameters characterising the thermodynamic cycle of the baseline engine and the optimum engines computed are shown in Fig. 2a. These same parameters, when expressed in relative terms using the characteristic parameters of the baseline engine, are illustrated in Fig. 2b. In turn, Figs. 2c, 2d and 2e show, respectively, the SFC, fuel burned and TET characterising each segment of the aircraft trajectory flown using the baseline and the three optimum engines determined. Finally, Fig. $2 \mathrm{f}$ shows a comparison of the main results associated with the three optimised engines determined in this first case study. As noticed in Fig. 2f, the three optimum engines computed led to (relatively) significant reductions in cruise SFC ( -8\%), fuel burned ( -7\%), and NOx emitted ( -70\%). From this figure, one can see as well that the variations in carbon dioxide and water vapour emissions are directly proportional to the variations in the amount of fuel burned (species in chemical equilibrium). This last situation repeats itself in all of the case studies analysed in this work.

The rate of fuel consumption in an aircraft engine (e.g. turbofan) is usually expressed in terms of SFC, which is defined as the engine fuel mass flow rate divided by the net thrust produced by the engine. For a given aircraft speed and fuel type, the SFC of a turbofan engine can be also expressed as being inversely proportional to both thermal efficiency and propulsive efficiency. It means that increases in these efficiencies result in reductions in SFC. Increases in both OPR and TET have a favourable effect on thermal efficiency. One way of reducing the SFC in turbofans involves then the simultaneous increase in OPR and TET. Regarding the engine BPR and FPR, it is well known that, for a given BPR, there is an optimum FPR which minimises SFC. For a given OPR, however, SFC decreases with the increase in the turbofan engine BPR. This is because as BPR rises, the optimum FPR decreases, so does in general the jet gas velocity, thereby yielding improvements in propulsive efficiency, and, consequently reductions in SFC. In the turbofan engines 
optimised in these case studies, however, it is not possible to increase BPR indefinitely because of the constraints imposed when defining the optimisation problem. Since the overall inlet air mass flow rate is fixed in this work, increases in BPR imply reductions in engine core mass flow rate. This, in turn, translates into higher OPR and/or TET values to cope with the higher work required from the core flow to move the larger amount of air that bypasses the core engine. As when OPR and TET are increased for better thermal efficiency, better compressor and turbine materials are required in these situations to cope with the increases in both CDT and TET. In addition, very high values of OPR and BPR will also require eventually small blades at the rear of the HPC.

Accordingly, as illustrated in Fig. 2a, the cruise SFC optimised engine is characterised by relatively high values of TET and OPR, which improves the engine thermal efficiency, and, consequently, reduces the engine cruise SFC. The results suggest that the BPR has been increased only to an extent in which the reduced core flow can cope with the work required by the fan under conditions of maximum OPR, which seems to have been established by restrictions in the maximum value of CDT (at TO) allowed in the process. Engine TET, in turn, seems to be a compromise between increasing its value - in order to augment the engine thermal efficiency and, hence, SFC - and reducing it in order to diminish propulsive efficiency degradations, and, consequently, increases in SFC. As expected, Fig. 2c shows that segments 4 and 5 exhibit the lowest SFC values. This is an obvious result once the cruise SFC optimisation involved the minimisation of a parameter computed averaging the SFC corresponding to these two segments considered as cruise in this work.

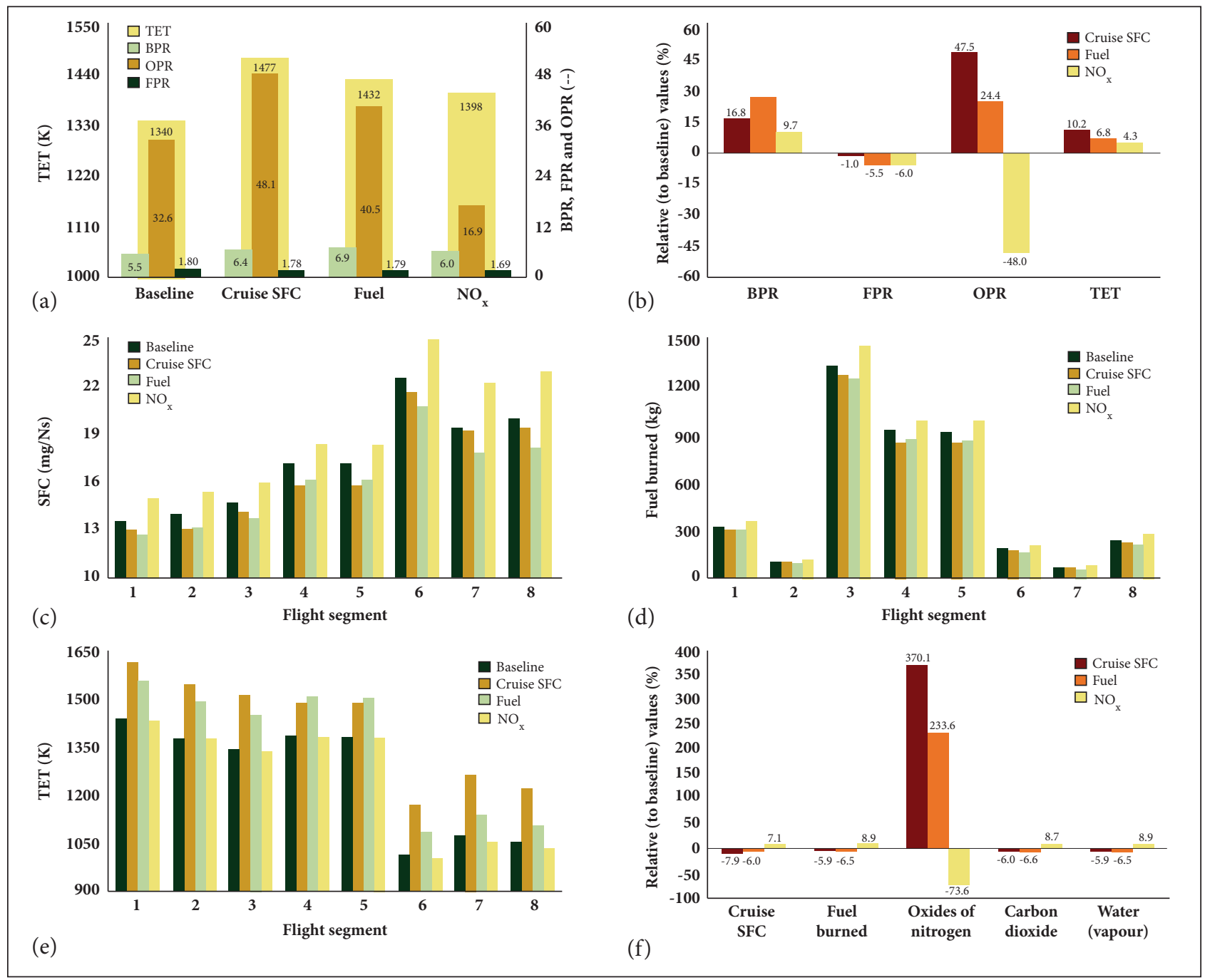

Figure 2. Case 1 - Two-Spool turbofan optimisation results. 
The fuel optimised engine results are similar to those ones obtained for the case in which the cruise SFC was minimised. This is expected, of course, once the objective functions are similar as well. The biggest difference between these two functions is that, when optimising for minimum fuel burned, the SFC corresponding to all trajectory segments is minimised and not only the SFC in cruise (as when optimising for minimum cruise SFC). Strictly speaking, minimisation of fuel burned implies minimisation of fuel mass flow rate (fixed flight profile and aircraft speeds, and hence segment flight times). However, due to SFC and fuel mass flow rate are directly related, minimisation of fuel burned can be also regarded as minimisation of SFC at all trajectory segments. Therefore, the main parameters driving the cruise SFC optimisation also play an important role when designing a turbofan engine for minimum fuel burned. Consequently, the fuel optimised engine is also characterised by relatively high values of OPR and TET (Fig. 2a). Regarding $\mathrm{BPR}$, the results show that, when optimising for minimum fuel burned, there is a further increase in BPR, which leads to improvements in propulsive efficiency, and, consequently, SFC reductions in almost all segments of the flight trajectory. Figure $2 \mathrm{~d}$ shows that the fuel optimised engine presents the lowest fuel consumption in all trajectory segments (except in cruise segments 4 and 5, where the cruise SFC optimised engine does), which yields, consequently, the lowest overall fuel burned.

There are four main parameters that affect $\mathrm{NO}_{\mathrm{x}}$ emissions which are directly or indirectly related to the engine operating conditions. These parameters are flame temperature, combustor air inlet temperature, air/fuel ratio and combustor air inlet pressure. Accordingly, a conventional engine optimised for minimum $\mathrm{NO}_{\mathrm{x}}$ emissions is expected to be characterised by both a relatively low combustor air inlet temperature (i.e. a low CDT and, consequently, a low OPR) and a relatively low combustor air inlet pressure (i.e. a low OPR). At the same time, the $\mathrm{NO}_{\mathrm{x}}$ optimised engine is expected to be operated using as low TET values as possible. All these aspects are confirmed by the results associated with the engine optimised for minimum $\mathrm{NO}_{\mathrm{x}}$ emissions. Thus, as observed in Fig. 2a, this engine presents the lowest OPR and TET of the three optimum engines computed. In addition, the engine BPR is slightly lower than those ones corresponding to the other two optimised engines. This allowed that the reduced OPR core flow provide the work demanded by the engine fan. As expected, the relatively low OPR and TET characterising the $\mathrm{NO}_{\mathrm{x}}$ optimised engine worsened its SFC. This is reflected in the high values of SFC and fuel burned characterising each aircraft trajectory segment. When compared to the other engines computed, the engine optimised for minimum $\mathrm{NO}_{x}$ presents the highest SFC (Fig. 2c) and fuel burned (Fig. 2d) values at each trajectory segment. Even so, the $\mathrm{NO}_{\mathrm{x}}$ optimised engine TET values at each flight segment are the lowest ones (Fig. 2e). This, of course, translated into low $\mathrm{NO}_{\mathrm{x}}$ emissions at each flight segment and, consequently, in the lowest overall $\mathrm{NO}_{\mathrm{x}}$ emissions characterising this optimised engine. On the contrary, the relatively high OPR and TET values characterising the cruise SFC and fuel optimised engines produce significant increases in $\mathrm{NO}_{\mathrm{x}}$ emissions and reductions in fuel burned (Fig. 2f). Similar analyses to those ones carried out in this first case study will be performed in the remaining case studies.

\section{CASE 2: THREE-SPOOL TURBOFAN OPTIMISATION}

Figure 3 illustrates the main results obtained when determining the optimum engines computed in this second case study. Since the same type of plots presented in Fig. 2 is included in this figure, a detailed description of Fig. 3 plots is not included in this section. Thus, as visualised in Fig. 3f, the cruise SFC optimised engine yielded only an small improvement in cruise SFC ( -2\%), while the other two optimum engines computed, similar to the twospool case, led to relatively significant reductions in fuel burned $(\sim-8 \%)$ and $\mathrm{NO}_{\mathrm{x}}$ emitted ( -95\%). In general, the three-spool engine optimisation results are similar to the corresponding two-spool ones. Thus, only the main features of the results obtained in this case study will be highlighted. As in the first case study, the three-spool engine optimised for minimum cruise SFC is characterised by relatively high values of TET and OPR (Fig. 3a). Since BPR increases generally lead to improvements in propulsive efficiency and, consequently, reductions in SFC, the BPR of the cruise SFC optimised engine is slightly higher than the baseline engine BPR.

In terms of OPR and TET, the fuel optimised engine is similar to the engine designed for minimum cruise SFC. Both are characterised by relatively high values of OPR and TET (Fig. 3a). As mentioned before, this is because the objective functions are similar as well. Similar to the two-spool case, a further increase in BPR is observed in the three-spool fuel optimised engine. This BPR increase yields improvements in propulsive efficiency and, consequently, SFC reductions in almost all segments of the flight trajectory. As illustrated in Fig. 3d, these results are reflected in the fuel that is burned at each flight segment. This figure shows that the fuel optimised 
engine presents the lowest fuel consumption in almost all trajectory segments and thus the lowest overall fuel burned.

All main features characterising two-spool engines optimised for minimum $\mathrm{NO}_{\mathrm{x}}$ also characterise three-spool ones. Thus, as verified in Fig. 3a, the $\mathrm{NO}_{\mathrm{x}}$ optimised engine presents the lowest OPR and TET of the three optimum engines computed. In addition, the engine BPR is lower than both the $\mathrm{BPR}$ values corresponding to the other two optimised engines and the baseline engine BPR. The considerable reduction in OPR seems to have been compensated by this BPR decrease, which allowed that the reduced OPR core flow be able to generate the work required for driving the engine fan. The significant reductions in OPR and TET observed in the case of the $\mathrm{NO}_{\mathrm{x}}$ optimised engine produced large increases in the engine SFC at each aircraft trajectory segment (Fig. 3c) and, consequently, in the corresponding fuel burned (Fig. 3d). The relatively low values of OPR, TET and BPR characterising the engine optimised for minimum $\mathrm{NO}_{\mathrm{x}}$ are therefore mainly responsible for the low levels of $\mathrm{NO}_{x}$ emitted at each flight segment, and, consequently, for the lowest overall $\mathrm{NO}_{\mathrm{x}}$ emissions that characterise this optimum engine (Fig. 3f).

\section{CASE 3: INTERCOOLED RECUPERATED TURBOFAN OPTIMISATION}

The results associated with the main parameters characterising the baseline and the optimum engines determined in the ICR engine case study are shown in Fig. 4. For details about the particular plots included in this figure, see the description of Fig. 2 plots. In particular, as observed in Fig. 4f, all three optimum engines

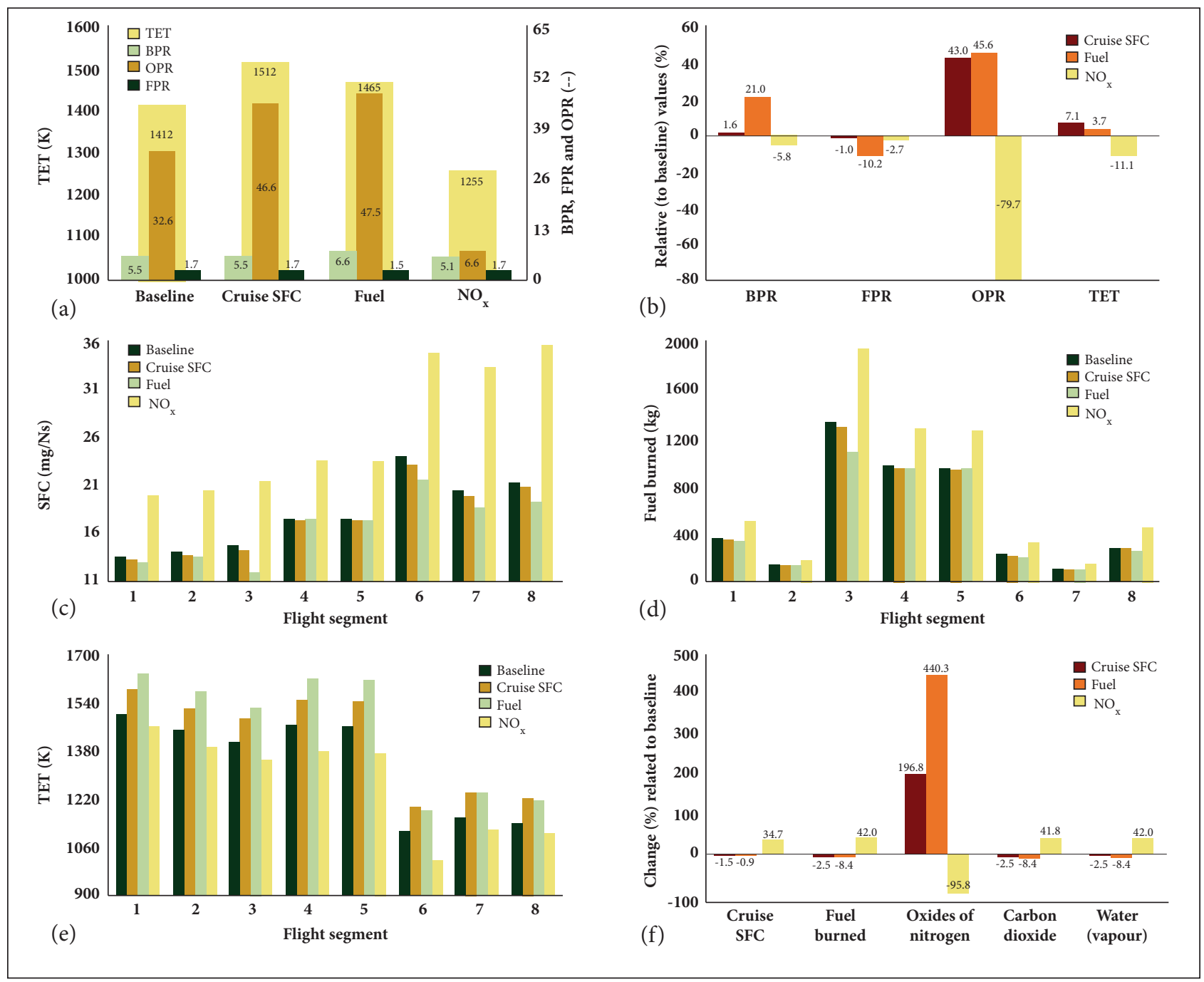

Figure 3. Case $2-$ Three-spool turbofan optimisation results. 
computed yield relatively significant reductions in cruise SFC ( - -12\%), fuel burned ( -14\%) and $\mathrm{NO}_{\mathrm{x}}$ emitted $(\sim-65 \%)$. Unlike conventional (two- or three-spool) engines and due to ICR engines offer higher efficiencies, and hence lower SFC values, with lower values of OPR (Saravanamuttoo et al., 2009; Boggia and Rüd, 2005), both the ICR engine optimised for minimum cruise SFC and the fuel optimised one are characterised by relatively high values of TET and only moderate OPR values (Fig. 4a). This is because these two factors contribute to the improvement of both engine thermal efficiency and effectiveness of the heat exchange processes and, consequently, to the reduction of SFC and fuel burned. TET seems to be a compromise between (i) increasing its value to improve thermal efficiency and heat exchange's effectiveness and, hence, SFC; and (ii) reducing it to avoid significant reductions in propulsive efficiency that can worsen SFC.
Figure $4 \mathrm{a}$ also shows that the BPR associated with the cruise SFC optimised engine is higher than the baseline engine BPR. An aspect that may have also a certain contribution to the increase in BPR is the reduction in compression work originated by both the reduction in OPR and the use of an intercooling process. When compared to the other optimum engines computed in this case study, the results show that the engine optimised for minimum fuel burned presents the highest BPR. These results are consistent with those associated with conventional engines (Figs. 2 and 3). The relatively high value of BPR used in the fuel optimised engine, together with the relatively high value of TET and moderate OPR (Fig. 4a), results in SFC reductions in almost all segments of the flight trajectory (Fig. 4c). This, of course, leads to the lowest overall fuel burned that characterises this ICR engine (Fig. 4f).

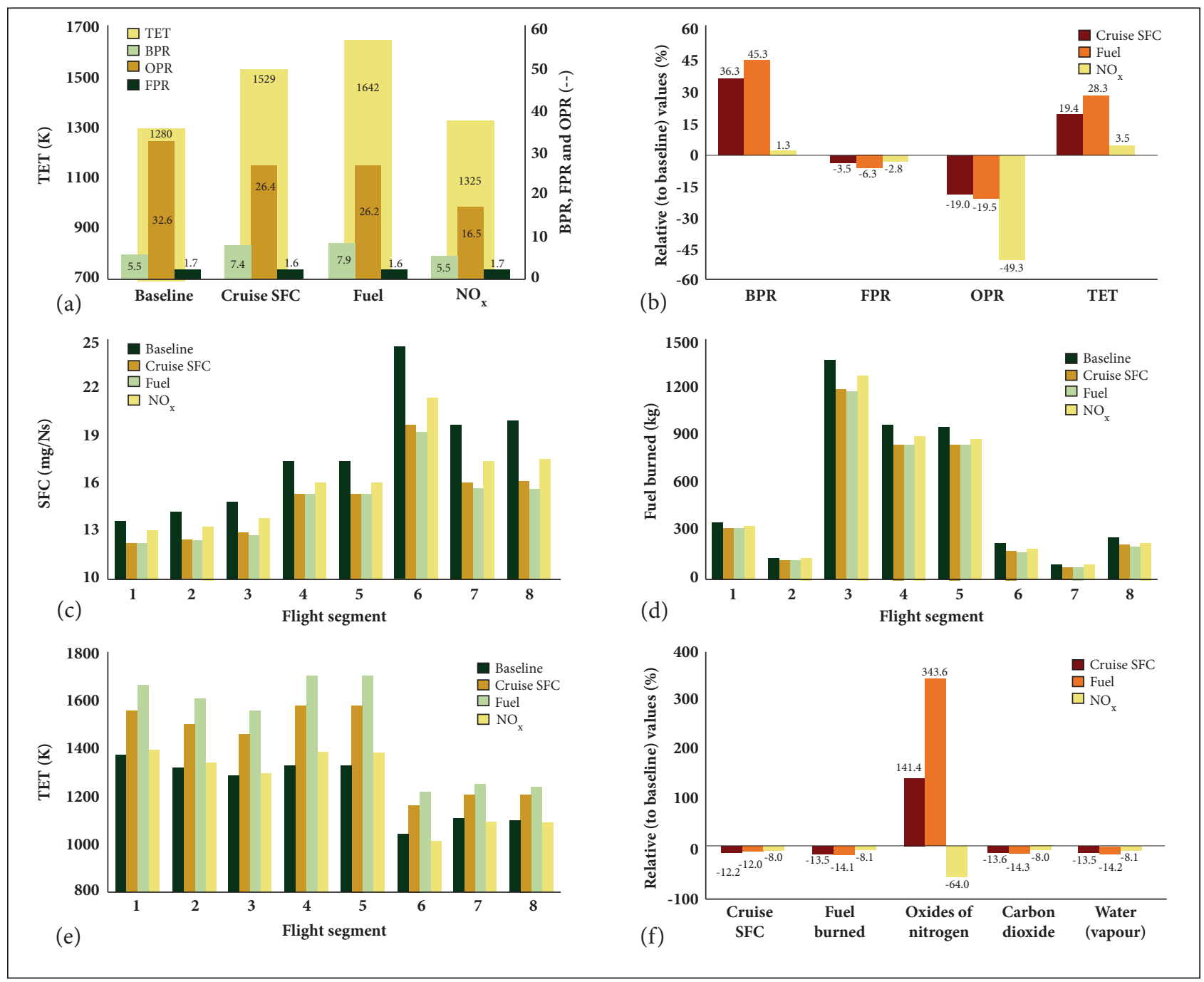

Figure 4. Case $3-$ Intercooled recuperated turbofan optimisation results. 
Conventional engines optimised for minimum $\mathrm{NO}_{x}$ emissions are expected to be characterised by relatively low values of combustor air inlet temperature, combustor air inlet pressure, and TET. In the case of ICR engines, air inlet temperature is mainly related to both CDT (and hence to OPR) and TET (through the recuperation system utilised). Air inlet pressure, in turn, is directly related to OPR. Accordingly, low values of combustor air inlet temperature and pressure mean low values of OPR and TET. Observing the results shown in Fig. 4a, it is possible to see that relatively low values of OPR and TET is indeed one of the characteristics of the ICR engine optimised for minimum $\mathrm{NO}_{\mathrm{x}}$ emissions. As shown in this figure, the $\mathrm{NO}_{\mathrm{x}}$ optimised engine presents the lowest OPR and TET of the three optimum engines computed. Regarding BPR, the results show that this parameter is roughly the same as in the case of baseline engine and lower than those values of BPR corresponding to the other two optimised engines. Similar to conventional engines, ICR engines optimised for minimum $\mathrm{NO}_{\mathrm{x}}$ emissions present relatively high SFC values at each aircraft trajectory segment (Fig. 4c), and cruise SFC and fuel optimised ones produce significant increases in $\mathrm{NO}_{\mathrm{x}}$ emissions (Fig. 4f).

\section{FURTHER RESULTS}

When analysing different engine cycles, it is important to find a means of comparing the different results obtained. This section describes therefore results showing a comparison of the three engine cycles analysed before. For this purpose, the baseline two-spool engine was considered as reference or baseline engine. For brevity, only one of the three objective functions usually utilised in the previous analyses, fuel, was used in the single-objective optimisation processes performed for comparison purposes. All other details associated with the optimisation of each particular engine configuration remained the same. As highlighted in the Engine Cycles section, the only difference related to the inclusion of the FN at DP and TO implicit constraints where required. The results of the comparison process of the three engine cycles are summarised in Fig. 5. In particular, the main cycle parameters associated with both the two-spool baseline engine and the three engine configurations optimised for minimum fuel burned are given in Fig. 5a. These same parameters, when expressed in relative terms, using the characteristic parameters of the two-spool baseline engine, are illustrated in Fig. 5b. In addition, Figs. 5c and $5 \mathrm{~d}$ show, respectively, the SFC characterising each segment of the aircraft trajectory, and the main results associated with the three optimised engines determined. As seen in Fig. 5d, the three-spool fuel optimised engine yielded only a relatively small reduction in the total amount of fuel burned $(\sim-2 \%)$, while the other two optimum engines computed led to relatively significant reductions in this parameter, i.e. two-spool -9\%, and ICR - $11 \%$. As expected, Fig. 5a illustrates that conventional (two- and three-spool) engines are characterised by relatively high values of OPR and TET. Unlike these results, the ICR engine is characterised by a moderate value of OPR. However, a relatively high TET value is also a characteristic of minimum fuel burned ICR optimised engines. As expected, the results (Fig. 5a) also show that, when compared to the two-spool baseline engine, the three optimum engines computed present higher values of BPR.

Comparing specifically the results corresponding to the three engine configurations optimised for minimum fuel burned, it is possible to see from Fig. 5a that the conventional engines present OPR and TET values which are roughly comparable. The relatively high TET value associated with the ICR engine seems to be related to its recuperation system which requires as high TET values as possible. The relatively low value of OPR characterising the ICR engine is, of course, a requirement of the intercooling and recuperation systems used in ICR engines. In terms of compression stages and cooling flow, it seems then that an ICR engine is "simpler" than conventional ones; but it also has, of course, all the other complexities involved when using the heat exchangers present in this type of engines. Looking at the overall results, the conventional three-spool engine optimised for minimum fuel, on one hand, presents the smallest reduction in fuel burned (Fig. 5d). Because of the simplifications introduced into the optimisation processes, it is not possible to determine with certainty the reasons behind the differences (in terms of fuel burned reduction) between the results obtained for the conventional two- and three-spool engines. Further optimisation work involving higher fidelity computational tools able to model, for instance, changes in engine weight and dimensions as the engine thermodynamic cycle changes should help to clarify this point. The ICR engine, on the other hand, yields the largest reduction in this parameter. This last result can be attributed to both the relatively lower SFC characterising an ICR engine and its improved part-load performance. Regarding $\mathrm{NO}_{\mathrm{x}}$ emissions, Fig. 5d shows that the ICR engine produces the smaller amount of this pollutant. The relatively low values of OPR characterising ICR engines seem to be partially responsible for this result. 


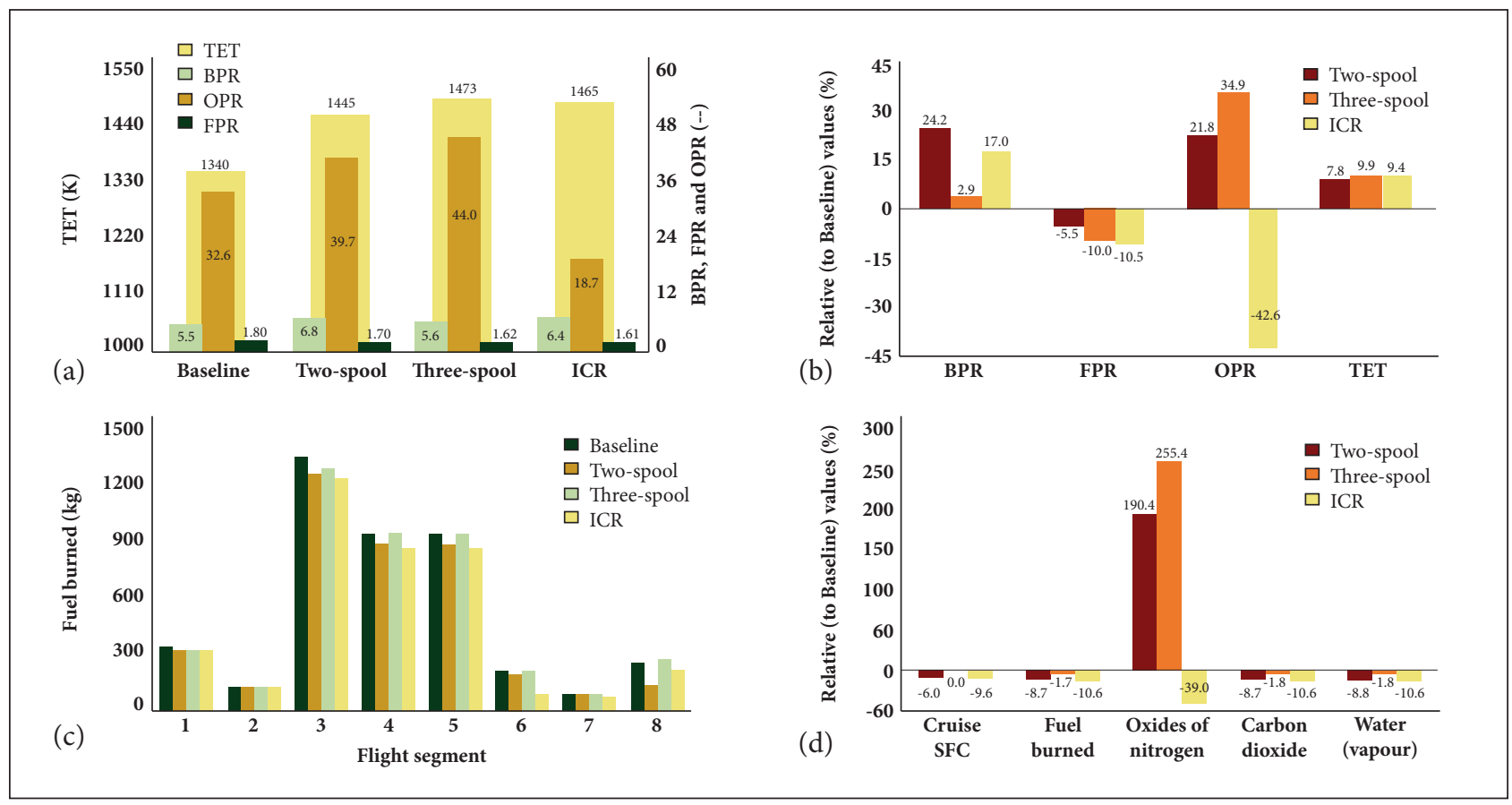

Figure 5. Comparison of engines - optimum engine cycles results.

\section{CONCLUSIONS}

In this work, the Techno-economic Environmental Risk Analysis (TERA) framework, an idea conceived and developed by Cranfield University, was utilised in conjunction with an in-house optimiser (i.e. Polyphemus) to carry out aircraft engine cycle optimisation processes. The optimisation processes focused primarily on the evaluation of the capabilities of Polyphemus for performing this type of optimisation processes. For the determination of optimum engine cycles then, simplifications were introduced into the optimisation processes when both defining the aircraft flight trajectory and modelling the different engine configurations analysed. Accordingly, several optimum engine cycles minimising separately three objective functions, cruise SFC, fuel burned, and $\mathrm{NO}_{\mathrm{x}}$ emitted, were determined. The optimum engine cycles results showed that conventional two- or three-spool engines optimised for minimum cruise SFC and fuel burned are characterised by relatively high values of TET, OPR, and BPR. Since these parameters directly influence the level of $\mathrm{NO}_{\mathrm{x}}$ emitted, conventional (two- or threespool) engines optimised for minimum $\mathrm{NO}_{\mathrm{x}}$ emissions are characterised by relatively low values of OPR and TET. ICR engines optimised for both minimum cruise SFC and minimum fuel burned are also characterised by relatively high values of
TET, but only moderate OPR values. Further reductions in OPR characterise minimum $\mathrm{NO}_{\mathrm{x}}$ emissions ICR optimised engines. Regarding BPR specifically, the ICR engine results obtained were consistent with the conventional engines ones. The engine cycle optimisation processes carried out yielded optimum results that reflect the general trends that could be expected when optimising according to the objective functions used in this work. It follows then that Polyphemus is suitable for carrying out this type of optimisation processes. This, of course, provides the necessary motivation for continuing with the development of the Polyphemus optimiser. As future work, in order to further elucidate the actual Polyphemus capabilities, optimum results obtained using this optimiser will be compared with known optimum solutions obtained using other well-accepted optimisers. It is expected then that Polyphemus be used in future for both determining optimum and "greener" aircraft engine cycles and optimising the preliminary design of this type of engines.

\section{ACKNOWLEDGEMENTS}

During this work, Cesar Celis was partially supported by the Programme Alßan, the European Union Programme of High Level Scholarships for Latin America, Scholarship 
No. E07D400097BR. The authors would like to extend their gratitude to both Mr. Richard Long, who developed the APM utilised, and Dr. Jean-Michel Rogero, whose PhD research at Cranfield University has provided a foundation for the Polyphemus optimiser used in this work. The financial support from the European Union is gratefully acknowledged.

\section{REFERENCES}

Antoine, N.E. and Kroo, I.M., 2005, "Framework for Aircraft Conceptual Design and Environmental Performance Studies", AIAA Journal, Vol. 43, No. 10, pp. 2100-2109.

Antoine, N.E., Kroo, I.M., Willcox, K. and Barter, G., 2004, "A Framework for Aircraft Conceptual Design and Environmental Performance Studies", Proceedings of the AIAA-2004-4314, 10th AIAA/ISSMO Multidisciplinary Analysis and Optimization Conference, Albany, USA.

Boggia, S. and Rüd, K., 2005, "Intercooled Recuperated Gas Turbine Engine Concept", Proceedings of the AIAA 2005-4192, 41st AIAA/ASME/SAE/ASEE Joint Propulsion Conference \& Exhibit, Tucson, USA.

Celis, C., 2010, "Evaluation and Optimisation of Environmentally Friendly Aircraft Propulsion Systems", Ph.D. Thesis, Cranfield University, Cranfield, United Kingdom.

Celis, C., Long, R., Sethi, V. and Zammit-Mangion, D., 2009a, "On Trajectory Optimisation for Reducing the Impact of Commercial Aircraft Operations on the Environment", Proceedings of the 19th Conference of the International Society for Air Breathing Engines, ISABE-2009, Montréal, Canada.

Celis, C, Moss, B. and Pilidis, P., 2009b, "Emissions Modelling for the Optimisation of Greener Aircraft Operations", Proceedings of the GT2009, ASME Turbo Expo 2009, Power for Land, Sea and Air, Orlando, USA.

Celis, C., Mohseni, M., Kyprianidis, K., Sethi, V., Ogaji, S., Haslam, A. and Pilidis, P., 2008, "Multidisciplinary Design Optimization of Aero Engines: Environmental Performance-based Methodology", Proceedings of the International Symposium on Compressor \& Turbine Flow Systems - Theory \& Application Areas, SYMKOM'08, Lodz, Poland.

Celis, C., Sethi, V., Zammit-Mangion, D., Singh, R. and Pilidis, P., 2014, "Theoretical Optimal Trajectories for Reducing the Environmental Impact of Commercial Aircraft Operations", Journal of Aerospace Technology and Management, Vol. 6, No. 1, pp. 29-42. doi: 10.5028/jatm.v6i1.288

Di Lorenzo, G., Friconneau, V., Brandt, P., Lonneux, V., Marinai, L., Pilidis, P. and Ruiz-Olalla, G., 2007, "Technoeconomic Environmental Risk Analysis - Technological Perspective Application to Low Carbon Plant", Proceedings of the 4th IDGTE Gas Turbine Conference 'Gas Turbine Technology in a Carbon Constrained World', Milton Keynes, United Kingdom.

Diedrich, A., Hileman, J., Tan, D., Willcox, K. and Spakovszky, Z., 2006, "Multidisciplinary Design and Optimization of the Silent Aircraft", Proceedings of the AIAA 2006-1323, 44th AIAA Aerospace Sciences Meeting, Reno, USA.

Goldberg, D.E., 1989, "Genetic Algorithms in Search, Optimization and Machine Learning", Addison-Wesley, Reading, USA.

Hartley, S.J., 1998, "Concurrent Programming: The Java Programming Language", Oxford University Press, New York, USA.
Khan, R., Barreiro, J., Lagana, M. C., Kyprianidis, K., Ogaji, S., Pilidis, P. and Bennett, I., 2009, "An Assessment of the Emissions and Global Warming Potential of Gas Turbines for LNG Applications", Proceedings of the GT2009, GT2009-59184, ASME Turbo Expo 2009, Power for Land, Sea and Air, Orlando, USA.

Kyprianidis, K., Au, D., Ogaji, S. and Grönstedt, T., 2009, "Low Pressure System Component Advancements and its Impact on Future Turbofan Engine Emissions", Proceedings of the 19th Conference of the International Society for Air Breathing Engines, ISABE-2009-1276, Montréal, Canada.

Kyprianidis, K.G., Di Lorenzo, G., Ogaji, S., Pilidis, P., 2008, "The TERA Approach - A Methodology for Techno-economical, Environmental and Risk Analysis of Multidisciplinary Systems", Cranfield University MultiStrand Conference, Cranfield, United Kingdom.

Long, R.F, 2009, "An Aircraft Performance Model for Trajectory Optimisation", Cranfield University, United Kingdom (unpublished).

Ogaji, S., Pilidis, P. and Hales, R., 2007, "TERA - A Tool for Aero-engine Modelling and Management", Proceedings of the 2nd World Congress on Engineering Asset Management and 4th International Conference on Condition Monitoring, Harrogate, United Kingdom.

Ogaji, S., Pilidis, P. and Sethi, V., 2009, "Advanced Power Plant Selection: The TERA (Techno-economic Environmental Risk Analysis) Framework", Proceedings of the 19th Conference of the International Society for Air Breathing Engines, ISABE-2009-1115, Montréal, Canada.

Ogaji, S., Sethi, V., Pilidis, P, Singh, R., Celis, C., Di Lorenzo, G., and Zammit-Mangion, D., 2010, "Power Plant Selection and Deployment: The TERA (Technoeconomic Environmental Risk Analysis]", Proceedings of the 5th European Turbine Network (ETN) International Gas Turbine Conference, The Future of Gas Turbine Technology, Brussels, Belgium.

Palmer, J.R., 1999, "The TurboMatch Scheme for Gas-Turbine Performance Calculations: User's Guide”, Cranfield University, Cranfield, United Kingdom.

Rogero, J.M. and Rubini, P.A., 2003, "Optimisation of Combustor Wall Heat Transfer and Pollutant Emissions for Preliminary Design Using Evolutionary Techniques", Proceedings of the Institution of Mechanical Engineers, Part A: Journal of Power and Energy, Vol. 217, No. 6, pp. 605-614. doi: 10.1177/095765090321700607

Saravanamuttoo, H.I.H., Rogers, C.F.G., Cohen, H. and Straznicky, P.V., 2009, "Gas Turbine Theory", 6th Edition, Pearson Prentice Hall, Harlow, England.

Whellens, M.W., 2003, "Multidisciplinary Optimisation of Aero-Engines Using Genetic Algorithms and Preliminary Design Tools", Ph.D. Thesis, Cranfield University, Cranfield, United Kingdom.

Wienke, D., Lucasius, C.B. and Kateman, G., 1992, "Multicriteria Target Vector Optimization of Analytical Procedures Using a Genetic Algorithm. Part I. Theory, Numerical Simulation and Application to Atomic Emission Spectroscopy", Analytica Chimica Acta, Vol. 265, No. २, pp. 211-225. doi: 10.1016/0003-2670(92)85027-4 